\title{
Cross-Species Transmission of Coronaviruses in Humans and Domestic Mammals, What Are the Ecological Mechanisms Driving Transmission, Spillover, and Disease Emergence?
}

\author{
Nicole Nova ${ }^{1}$ \\ ${ }^{1}$ Department of Biology, Stanford University
}

September 15, 2021

\begin{abstract}
Coronaviruses cause respiratory and digestive diseases in vertebrates. The recent pandemic, caused by the novel severe acute respiratory syndrome coronavirus 2, is taking a heavy toll on society and planetary health, and illustrates the threat emerging coronaviruses can pose to the wellbeing of humans and other animals. Coronaviruses are constantly evolving, crossing host species barriers, and expanding their host range. In the last few decades, several novel coronaviruses have emerged in humans and domestic animals. Novel coronaviruses have also been discovered in captive wildlife or wild populations, raising conservation concerns. The evolution and emergence of novel viruses is enabled by frequent cross-species transmission. It is thus crucial to determine emerging coronaviruses' potential for infecting different host species, and to identify the circumstances under which cross-species transmission occurs in order to mitigate the rate of disease emergence. Here, I review (broadly across several mammalian host species) up-to-date knowledge of host range and circumstances concerning reported cross-species transmission events of emerging coronaviruses in humans and common domestic mammals. All of these coronaviruses had similar host ranges, were closely related (indicative of rapid diversification and spread), and their emergence was likely associated with high-hostdensity environments facilitating multi-species interactions (e.g., shelters, farms, and markets) and the health or wellbeing of animals as end- and/or intermediate spillover hosts. Further research is needed to identify mechanisms of the cross-species transmission events that have ultimately led to a surge of emerging coronaviruses in multiple species in a relatively short period of time in a world undergoing rapid environmental change.
\end{abstract}

\section{Hosted file}

Nova_2021.pdf available at https://authorea.com/users/330225/articles/524506-cross-speciestransmission-of-coronaviruses-in-humans-and-domestic-mammals-what-are-the-ecologicalmechanisms-driving-transmission-spillover-and-disease-emergence 